\title{
Nationalstaaten und Europäische Union - historische Vorbilder für eine staatspolitische Innovation?
}

von Dieter Langewiesche

Will man die Europäische Union nicht als Gebilde „sui generis “ betrachten und sich so der begrifflichen Einordnung entziehen, liegt es nahe, nach historischen Vorbildern zu suchen. In diesem Rahmen sind insbesondere der Nationalstaat und die historischen Bünde, ob im Spannungsfeld von Staatenbund und Bundesstaat oder als eigenständige Ordnungsform, in die Diskussion eingebracht worden. Gegen den Nationalstaat als historisches Vorbild spricht jedoch nicht nur das Fehlen notwendiger Voraussetzungen, sondern auch die Tatsache, dass dessen Errichtung immer mit Krieg und Gewalt verbunden war. Mit Blick auf die historischen Bünde bestehen ebenfalls fundamentale Unterschiede zur gegenwärtigen Situation der EU, wie das Beispiel des Deutschen Bundes verdeutlicht.

If one rejects the classification of the EU as a structure "sui generis", it seems obvious to look for historical precedents. Especially the nation-state and historic confederations are objects of discussion. Nevertheless, the EU lacks essential pre-conditions for forming a nation-state, and furthermore the formation of nation-states was always accompanied by war and violence. Regarding the model of the confederations, the example of the $19^{\text {th }}$ century's German Confederation shows fundamental differences to the present European integration.

Bietet die Staatengeschichte Vorbilder für die EU, die es ermöglichen, diese „größte Erfindung unserer Zeit" ${ }^{\text {*1 }}$, angemessen zu verstehen und ihre Entwicklungspotentiale vor dieser geschichtlichen Folie einzuschätzen? Wer diese Frage bejaht, pflegt die institutionelle Integration Europas in Analogie zu drei Staatsmodellen zu betrachten, die sich in den vergangenen Jahrhunderten identifizieren lassen: (a) der Nationalstaat - vor allem in seiner föderativen Gestalt, (b) Staatenbund versus Bundesstaat - ein Spannungsfeld mit gleitenden Übergängen, (c) der Bund als eigenständige, historisch erprobte Ordnung, die sich sowohl der

1 Grimm, D.: Die größte Erfindung unserer Zeit, in: Frankfurter Allgemeine Zeitung v. 16. 06. 2003. 
gängigen Alternative Staatenbund/Bundesstaat als auch dem Leitbild Nationalstaat entziehe.

\section{Nationalstaat und Nation - historische Analogie für die Zukunft der EU?}

Die Orientierung am Nationalstaat, der sich im 19. Jahrhunderts und verstärkt nach dem Zweiten Weltkrieg angesichts der militärisch erzwungenen Auflösung der multinationalen Reiche (Habsburgermonarchie und Osmanisches Reich) als staatliches Leitbild allgemein durchzusetzen schien ${ }^{2}$, bietet offensichtlich keine Hilfen, den europäischen Integrationsprozess und die Institutionenordnung, die er bislang ausgebildet hat, vergleichend zu analysieren. Dennoch wird er als Vergleichsmaß immer wieder angelegt, sei es erwartungsfroh oder abwehrend. Die Entstehung von Nationalstaaten war in der Vergangenheit jedoch an Voraussetzungen gebunden, die auf europäischer Ebene nicht vorhanden sind, und sie verlief in Bahnen, die niemand einschlagen will.

Um mit dem Unerwünschten zu beginnen: Nahezu alle Nationalstaaten sind Kriegsgeschöpfe, hervorgegangen aus Staatenkriegen oder Revolutionskriegen, oft verbanden sich beide. ${ }^{3}$ Die Entstehung aus der Kriegsgewalt ist keine historische Besonderheit des Nationalstaates. Er steht hier in einer langen Tradition, die er jedoch radikalisiert hat. Der amerikanische Historiker Charles Tilly hat sie auf die griffige Formel gebracht: „War made the state, and the state made war. “4 Für das 19. Jahrhundert könnte man sinngemäß fortsetzen: Der Krieg macht den Nationalstaat, der Nationalstaat macht Krieg, um außerhalb Europas zum Impe-

2 Dass die multinationalen und multiethnische Reiche oder Imperien jedoch trotz der Orientierung am Leitbild Nationalstaat nie ihre Bedeutung verloren haben, betonen zu recht Osterhammel, J.: Die Verwandlung der Welt. Eine Geschichte des 19. Jahrhunderts, München, 2009; Darwin, J.: After Tamerlane. The Rise and Fall of Global Empires, 1400-2000, London, 2007; s. auch Leonhard, J./Hirschhausen, U.v.: Empires und Nationalstaaten im 19. Jahrhundert, Göttingen, 2009; Langewiesche, D.: Das Alte Reich nach seinem Ende. Die Reichsidee in der deutschen Politik des 19. und 20. Jahrhunderts. Versuch einer nationalgeschichtlichen Neubewertung in welthistorischer Perspektive, in: ders.: Reich, Nation, Föderation. Deutschland und Europa, München, 2008, 211-234.

3 Ausführlich dazu Langewiesche, D.: Nation, Imperium und Kriegserfahrungen, in: Schild, G./Schindling, A. (Hg.): Kriegserfahrungen - Krieg und Gesellschaft in der Neuzeit: Neue Horizonte der Forschung, Paderborn, 2009, i. E.; ders.: Fortschrittsmotor Krieg. Krieg im politischen Handlungsarsenal Europas im 19. Jahrhundert und die Rückkehr der Idee des bellum iustum in der Gegenwart, in: Benninghaus, C. u. a. (Hg.): Unterwegs in Europa. Beiträge zu einer vergleichen Sozial- und Kulturgeschichte, Frankfurt/M., 2008, 23-40.

4 Tilly, C.: Reflections on the History of European State-Making, in: ders. (Hg.): The Formation of National States in Western Europe, Princeton, 1975, 3-83, hier 42. 
rium zu expandieren. Den Zeitgenossen des 19. Jahrhunderts war diese Symbiose von Nationalstaat und Krieg bewusst. Es genügt, an Ernest Renan zu erinnern, der gern zitiert wird, wenn man die Nation als Demokratisierungskraft aufruft: die Nation - ,un plébiscite de tous les jours “. In seinem berühmten Vortrag von 1882 „Qu'est-ce qu'une nation?“, aus dem dieses Zitat stammt, rechnete es Renan zu den Kardinalfehlern des Hauses Habsburg, die Ungarn und die Slawen, die Tschechen und die Deutschen nicht zu einer einzigen Nation staatlich verschmolzen zu haben, notfalls, so Renan, als ,le résultat d'une extermination et d'une terreur" wie bei der Vereinigung Frankreichs (,,la réunion de la France du Nord et de la France du Midi"). ${ }^{5}$

Dieses Vertrauen in den Fortschritt durch Krieg und Gewalt ist auch der Gegenwart nicht gänzlich fremd; ${ }^{6}$ früheren Zeiten war sie vertraut: kein Nationalstaat ohne Krieg, keine erfolgreiche Nationalrevolution ohne Krieg, keine Ausweitung des Nationalstaates zum Imperium ohne Krieg. Dieses Erfahrungswissen hatten die Menschen im 19. und bis weit ins 20. Jahrhundert vor Augen, und ihr Geschichtswissen bestätigte es. Den Krieg für höchste Ziele einzusetzen, galt ihnen als notwendig. Im Nationalstaat, vor allem im machtvoll großen, sahen sie einen Letztwert. Historiker wie der Schweizer Jacob Burckhardt oder der Niederländer Johan Huizinga haben in ihrem Lob des bürgernahen Kleinstaates vergeblich gewarnt vor dieser Fixierung auf machtvolle Größe, die der Gestaltung durch den Bürger entgleite.

Die Entstehung der Nationalstaaten lässt sich historisch drei Typen zuordnen, und alle waren mit dem Einsatz von Kriegsgewalt verbunden. ${ }^{7}$

(a) Ein bestehender Staat wird in einen Nationalstaat transformiert. Der Nationalstaat ist hier der Erbe eines jahrhundertelangen Staatsbildungsprozesses, den er mächtig vorantrieb. Staatsbildung meinte in der Vergangenheit immer auch, den Staat institutionell vereinheitlichen und die Staatsgewalt zentralisieren. Auf

5 Renan, E.: Qu'est-ce qu'une nation?, in: Euvres Complètes de Ernest Renan, hg. v. H. Psichari, 2 Bde., Paris, 1947, Bd. 1, 887-906, hier 904, 891.

6 Langewiesche, D.: Fortschrittsmotor Krieg, a. a. O.

7 Diese Typisierung modifiziert Begriffe Theodor Schieders und Michael Manns. Schieder, T.: Nationalismus und Nationalstaat. Studien zum nationalen Problem im modernen Europa, hg. v. Dann, O./Wehler, H.-U., 2. Aufl., Göttingen, 1992, unterschied zwischen dem integrierenden Nationalstaat des westeuropäischen Typs, dem unifizierenden Nationalstaat (Deutschland, Italien) und dem sezessionistischen Nationalstaat (hervorgehend aus übernationalen Imperien wie der Habsburgermonarchie). Mann, M.: The Sources of Power. Vol. II: The Rise of Classes and Nation-States, 1760-1914, Cambridge, 1993, 218, differenziert zwischen ,nation as state-reinforcing“ (vor allem Frankreich und Großbritannien), ,state-creating“ (Preußen-Deutschland) und ,,state-subverting “ (Habsburgermonarchie). 
diesem Weg waren die Fürsten in der frühen Neuzeit unterschiedlich weit vorangekommen. Wo seit dem ausgehenden 18. Jahrhundert die Transformation des frühneuzeitlichen Fürstenstaats in einen modernen Nationalstaat gelang, gewann die Zentralisierung der Staatsgewalt und die institutionelle Vereinheitlichung des Staates eine enorme Schubkraft. Die Idee der Nation zielt auf eine rechtlich und politisch homogene Staatsbürgergesellschaft, und der Nationalstaat erwies sich überall als die mächtigste Kraft auf diesem Weg. Der Nationalstaat verfuhr dabei weitaus rigider als der alte Fürstenstaat. In Frankreich zentralisierte die revolutionäre Nation mit einer Gewalt, die dem absolutistischen Monarchen nicht zur Verfügung stand. Die Nation und der Nationalstaat wirkten als Zwingherren der Zentralisierung staatlicher Funktionen und der Homogenisierung der Staatsbürgergesellschaft.

(b) Ein Nationalstaat entsteht, indem er mehrere Staaten vereinigt. Die Nationalstaatsgründungen in Deutschland und Italien gehören zu diesem unifizierenden Typus. Beiden gemeinsam war, dass sie nicht nur Staaten vereinten, sondern zugleich trennten. Unifizierung und Sezession geschah in einem einzigen Akt, ohne Sezession keine Vereinigung.

(c) Den dritten Typus - Nationalstaatsgründung durch Sezession - findet man dort, wo aus multinationalen Reichen Gebiete herausgesprengt werden. Nationalstaatsgründung bedeutet dann immer auch Staatszerstörung. Das Osmanische Reich etwa verlor im 19. und frühen 20. Jahrhundert von Krieg zu Krieg seine Territorien im südöstlichen Europa, und es entstanden Staaten, die sich selber als Nationalstaaten verstanden. Ich formuliere das so vorsichtig, weil die Kategorie Nation hier sehr problematisch ist und die Grenzziehung unter diesen neuen Staaten heftig umkämpft war, weil sie um Territorien konkurrierten, die sich national bzw. ethnisch nicht eindeutig zuordnen ließen. Staatszerstörung begleitete aber auch die Gründung des deutschen und des italienischen Nationalstaates, denn es mussten die staatlichen Bindungen zur Habsburgermonarchie durchtrennt werden. Auch das ging nicht ohne Krieg. Als Sezessionsstaat entstand auch der belgische Nationalstaat. Und die Entstehung der Nationalstaaten im Norden Europas lässt sich ebenfalls nur als eine Kette fast immer gewaltsamer Sezessionen verstehen.

Der europäische Integrationsprozess und dessen Institutionalisierung zielen darauf, mit dieser Kriegsgeschichte zu brechen. In diesem Sinne setzt die EU den Willen zum Ausstieg aus einer Hauptlinie der europäischen Geschichte voraus, und nicht nur der europäischen. Das geschichtliche Europa hatte seine staatliche Gestalt stets mit den Mitteln des Krieges geformt. Die kulturellen Gemeinsam- 
keiten Europas seit der Antike stellten diese Bereitschaft, den Krieg als Instrument der Staatsbildung einzusetzen, nie in Frage. Das geschah erstmals im europäischen Integrationsprozess der Gegenwart. ${ }^{8}$

Wer im Nationalstaat und seinen Entstehungsgeschichten ein historisches Vorbild für Staatsbildungsprozesse auf europäischer Ebene sieht, verdunkelt dieses Zentralereignis in der europäischen Geschichte nach dem Zweiten Weltkrieg: Die Bereitschaft von Staaten, in einem offenen Prozess, dessen Verlauf nicht entlang historischer Erfahrungen eingeschätzt werden kann, eine Institutionenordnung zu schaffen, die Gemeinschaftsinteressen formuliert und sie gegenüber den Mitgliedsstaaten notfalls mit Entscheidungen des Europäischen Gerichtshofes durchsetzt, trennt die EU scharf von der Geschichte aller Nationalstaaten. Die Vergangenheit bietet nichts, das sich damit vergleichen ließe.

Auch wenn man den Zeitrahmen erweitert und die Prozesse der Nationsbildung betrachtet, die der Gründung des Nationalstaates vorausgingen, wird man vornehmlich Unterschiede zur heutigen Situation beobachten. Es gibt jedoch auch Analogien. Betrachtet man die innere Nationsbildung als einen Prozess, der sich auf drei miteinander verschränkten Ebenen vollzieht, so lassen sich im EU-Raum durchaus Entwicklungen feststellen, die in früheren Zeiten innerhalb der Nationalstaaten und ihrer Vorgänger verlaufen sind. Am weitesten sind diese Entwicklungen, die darauf zielen, einen Raum mit homogenen Strukturen zu schaffen, in den Bereichen Wirtschaft und Recht vorangeschritten, in erheblich geringerem Maße bei gesellschaftlichen Organisationen wie Parteien, Gewerkschaften oder Kirchen und am wenigsten auf der Ebene von Kultur und Mentalität. Immerhin zeigen die Umfrageergebnisse, die im Eurobarometer veröffentlicht werden, dass viele Menschen in ihrem Selbstbild eine doppelte Loyalität ausgebildet haben: Sie fühlen sich als Mitglied ihrer Nation und Bürger ihres Nationalstaates, aber auch als Bürger der EU.

In der Vergangenheit haben Doppel- oder Mehrfachloyalitäten dieser Art nur dann gleichberechtigt nebeneinander bestehen können, sofern nicht eine von ihnen den Vorrang vor anderen beanspruchte. So bald dies geschah, waren gewaltsame Konflikte unausweichlich. Die Nation kam dann mit ihrer Forderung nach demokratischer „Selbstregierung der Völker“ stets im „Kampfwagen“ daher, wie der Austromarxist Otto Bauer bereits 1907 hellsichtig diagnostiziert

8 Das habe ich näher ausgeführt in: Staat, Nation und Föderation in der europäischen Geschichte, hg. v. Gerda Henkel Stiftung, Münster, 2008; Historische Wege nach Europa, in: Langewiesche, D.: Nation, Nationalismus, Nationalstaat in Deutschland und Europa, München, 2000, 217-230. 
hatte. ${ }^{9}$ Dass bestehende Nationalstaaten freiwillig und vertraglich geregelt Souveränitätsrechte abgeben, um sich einer supranationalen Institutionenordnung einzufügen, hat in der Vergangenheit nirgendwo einen Vorläufer. Ohne Vorbild wäre auch die Entstehung einer Nation ohne die gemeinsame Kriegserfahrung. Denn auch die innere Nationsbildung war historisch stets auf die emotionale Kraft angewiesen, die von Kriegen, gewonnenen und verlorenen, ausgehen. Kulturelle Gemeinsamkeiten allein reichten nirgendwo aus, eine Nation zu formen. Angesichts dieser historischen Erfahrung wäre es äußerst riskant, die Zukunft der EU in Analogie zur Nations- und Nationalstaatsbildungen in der Vergangenheit zu entwerfen und die Politik auf dieses Ziel auszurichten.

\section{Staatenbund/Bundesstaat versus Bund: die Geschichte als theoretische Rüstkammer für die Instutionenanalyse der EU?}

In der gegenwärtigen rechtswissenschaftlichen Diskussion dominieren, soweit sie in historischer Perspektive geführt wird, zwei Positionen bei der Analyse der institutionellen Gestalt der EU. Beide suchen nach Kontinuitäten, um das Neue zu verstehen. Dies soll an einigen konträr wertenden Studien erläutert werden.

Unter den jüngeren deutschen Rechtwissenschaftlern sind Christoph Möllers und Christoph Schönberger mit ambitionierten historisch-systematischen Deutungen hervorgetreten. Möllers nennt in seiner kritischen Analyse der Staatstheorie in der Bundesrepublik die häufige anzutreffende Charakterisierung der EU als ein Phänomen sui generis eine „,begriffliche Bankrotterklärung“(10. Als tragfähiges Begriffsfundament für einen strukturellen Rechtsvergleich schlägt er zweierlei vor: (a) das „Mehrebenen-System“ - einen politikwissenschaftlicher Ansatz, der viele Vorzüge hat, aber $\mathrm{m}$. W. bislang ohne historische Dimension geblieben ist und deshalb hier nicht weiter verfolgt wird; und (b) den „Bund“.

Die hohe Erwartung, die manche Juristen auf der Suche nach einer historischsystematischen Erklärung der EU an die Kategorie „Bund“ richten, hat programmatisch Christoph Schönberger ausgeführt. ${ }^{11}$ Indem er auf deren lange

9 Zit. n. Langewiesche, D.: „Die Sozialdemokratie hält die Nation für unzerstörbar und für nicht zerstörenswert“. Theoretische Reflexionen im Austromarxismus um 1900 und ihre Bedeutung für die heutige Nationalismusforschung, in: ders.: Reich, Nation, Förderation, a. a. O, 93-110, hier 94; vgl. ders.: Nationalismus als Pflicht zur Intoleranz, in: ebd., 53-67.

10 Möllers, C.: Der vermisste Leviathan. Staatstheorie in der Bundesrepublik, Frankfurt/M., 2008, 88; dort auch das Folgende.

11 Schönberger, C.: Die Europäische Union als Bund. Zugleich ein Beitrag zur Verabschiedung des Staatenbund-Bundesstaat-Schemas, in: Archiv des öffentlichen Rechts, 129/1 (2004), 81-120. 
Geschichte zurückgreift, will er das staatstheoretische Denken aus dem Dilemma führen, alle Formen von Staatlichkeit nur vor der Alternative Staatenbund oder Bundesstaat betrachten zu können. Gefangen in dieser begrifflichen Zwangsjacke lasse sich das Neue an der EU nur beschreiben als ,staatsanaloger Nichtstaat ${ }^{\star 12}$. Ebenso werde ihr föderaler Charakter verzeichnet, wenn sie nur in Analogien zum klassischen Bundesstaat gesehen werden. Diese Mängel einer gängigen rechtswissenschaftlichen Betrachtung der EU spitzt Schönberger zu einer sprachlichen Paradoxie zu: der „staatsanaloge Nichtstaat“ erscheine als ein

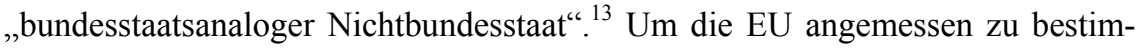
men, brauche es jedoch für "derartige Formen föderaler Verdichtung“ eines „eigenständigen Analyserasters“, und dieses biete die Theorie des Bundes. ${ }^{14} \mathrm{Sie}$ gebe es in systematischer Form noch nicht, doch das das Ziel der künftigen Rechtsystematik lasse sich benennen: der „Bund der Europäischen Union“15.

Schönberger und Möllers vergewissern sich, wenn sie den Bund für eine rechtssystematische Bestimmung der EU nutzen wollen, in der Geschichte. Und in der Tat, der Bund ist ein Begriff mit einer langen Vergangenheit. Reinhart Koselleck nannte ihn „einen Grundbegriff der menschlichen Verfassungsgeschichte“16, allerdings, so fügt er als Historiker des Begriffswandels hinzu, ein Begriff, der sich ,in leere Allgemeinheit verflüchtigt“”, wenn er nicht für den jeweiligen historischen Augenblick inhaltlich präzise gefüllt wird. Welch enormes Bedeutungsspektrum dieses Wort Bund historisch ausgefüllt, wie es sich entwickelt hat, was im Laufe der Zeit an Bedeutungen verloren ging und welche neu hinzukamen, vor allem - wie umkämpft diese Bedeutungen jeweils waren, führt Koselleck eindrucksvoll vor Augen. Der Begriff Bund bietet, betrachtet man seine Geschichte, also nichts, was man heute als Begriffsfundament für die rechtswissenschaftliche Gegenwartsbestimmung einfach übernehmen könnte. Wenn Juristen mit dem Begriff Bund das Phänomen EU erfassen und ihm eine historische Vergleichsdimension geben wollen, so können sie das nur, indem sie eine Begriffslinie auswählen, die aber immer eine politisch umkämpfte gewesen ist, in Konkur-

12 Ebd., 84.

13 Ebd.

14 Ebd., 85.

15 Ebd., 119.

16 Koselleck, R.: Bund, Bündnis, Föderalismus, Bundesstaat, in: Brunner, O./Conze, W./Koselleck, R (Hg.).: Geschichtliche Grundbegriffe, Bd. 1, Stuttgart, 1972, 582-671, hier 582; dort auch das folgende Zitat. 
renz zu vielen anderen Deutungen, mithin alles andere als ein historisch festes Fundament für den klärenden Vergleich, um die EU besser zu verstehen.

Warum ich bezweifle, dass ein Vergleich mit historischen Bünden das Neue an der EU erkennen lässt, werde ich im folgenden Abschnitt erörtern. Zuvor sollen noch weitere rechtswissenschaftliche Deutungen betrachtet werden.

Josef Isensee, Repräsentant einer älteren Juristengeneration mit anderen wissenschaftspolitischen Einstellungen, wie Möllers scharf betont ${ }^{17}$, richtet seinen rechtswissenschaftlichen Blick in anderer Perspektive auf die EU als Möllers und Schönberger, und deshalb wählt er aus dem historischen Arsenal der Begriffe für Staatskonstruktionen andere aus, mit denen er das Besondere der EU erklären will. Er spricht vom „Staatenverein der EU“18. Auch dieser Begriff ist ein historischer. Darauf geht Isensee nicht ein. Für ihn ist dieser Begriff nützlich, weil er das Fluide an der EU betont und damit hilft, das vertragliche Regelwerk, das die Mitgliedsstaaten der EU gegeben haben, in den Hintergrund zu rücken. Bei einem Juristen mag diese vertragsabgewandte Argumentation überraschen. „Ein Verein gewinnt sein Profil weniger über die Regeln seiner Satzung als durch die Personen seiner Mitglieder. Nicht anders der Staatenverein der EU. Mit jedem neuen Mitglied wandelt sich sein Charakter." ${ }^{\text {19 }}$

Die Geschichte seines Begriffs zur Definition der EU könnte Isensee Argumentationshilfe bieten. Denn im frühen 19. Jahrhundert kannten die Fachleute dieses Wort Staatenverein auch schon. Sie verwendeten es, bezogen auf die damaligen überstaatlichen Bünde, in der gleichen Absicht wie Isensee für die EU. Sie charakterisierten nämlich mit diesem Wort die Bünde deutscher Staaten, zunächst den Rheinbund, dann den Deutschen Bund, als unfähig und auch nicht darauf angelegt, die Souveränität der Einzelstaaten aufzuzehren. ${ }^{20}$ Eine solche Geschichtshilfe kann Isensee allerdings nicht brauchen, denn der ,Staatenverein der EU“ entzieht sich in seiner Sicht historischen Vergleichen durch, so sagt er, Originalität und Mutabilität.

17 Möllers, C., a. a. O., 63-67, zum von Isensee und Paul Kirchhof hrsg. „Handbuch des Staatsrechts der Bundesrepublik Deutschland“ (9 Bde.): ,eher als das Denkmal einer wissenschaftlichen und politischen Generation denn als staatstheoretischer Entwurf von Interesse“, 67.

18 Isensee, J.: Europäische Nation? Die Grenzen der politischen Einheitsbildung Europas, in: Decker, F./Höreth, M. (Hg.): Die Verfassung Europas, Wiesbaden, 2009, 254-280, hier 274.

19 Ebd.

20 Koselleck, R., a. a. O., 655-658. 
Stoppschild auf dem Weg zur vollen Staatlichkeit, darin liegt für Isensee die Bedeutung des Begriffs Staatenverein; und das meinte auch Paul Kirchhof, als er den Begriff Staatenverbund erfand, mit dem unter seiner Federführung das deutsche Bundesverfassungsgericht 1993 in einem vielbeachteten Urteil die EU bedachte. $^{21}$

Vom Staatenverbund spricht auch Dieter Grimm, auch er ehemaliger Verfassungsrichter, doch er begründet seine Warnung vor einer Verstaatlichung und Parlamentarisierung der EU nicht rechtsdogmatisch, sondern demokratietheoretisch. Er fragt nach der Demokratiefähigkeit der EU. Seine Antwort: Sie ist es noch nicht, und wenn man die Gründe akzeptiert, die er anführt, dann ist auch in Zukunft nicht abzusehen, wie in der EU die intermediären Strukturen entstehen könnten, von denen die Demokratie lebt. Eine EU-Verfassung im vollen Sinn könnte diese Strukturen nicht aus sich heraus schaffen. Sie wäre ein institutioneller Vorgriff, der die politische Realität verfehlte. Für die „größte Erfindung unserer Zeit‘ ${ }^{\star 22}$ sei deshalb der Vertrag, nicht die Verfassung die angemessene Grundlage. ${ }^{23}$

\section{Warum die historischen Bünde keine institutionellen Vorläufer der EU sind}

Die Europäische Union als die ,größte Erfindung unserer Zeit“, diese Einschätzung bestätigt sich in historischer Perspektive. Die EU ist dabei, etwas zu verwirklichen, wozu es in der Geschichte keine Parallelen und keine Vorläufer gibt: freiwilliger Verzicht bestehender Staaten auf wachsende Teile ihrer Souveränität. Aus dieser Sicht werde ich nun die Versuche zu staatlichen Neuordnungen in den letzten Jahrhunderten blicken - in Europa und vor allem im deutschsprachigen Raum, in dem die historische Tradition der Bünde besonders stark ausgeprägt war. Gefragt wird nach der Fähigkeit und Bereitschaft von Staaten, erhebliche Teile staatlicher Souveränität dauerhaft auf eine suprastaatliche Institutionenebene zu übertragen. Denn darin liegt das historisch Neue an der EU. Gibt es dafür Vorläufer in der Geschichte? Lassen sich die historischen Bünde als Vorläufer

21 Vgl. zu den Diskussionen und den Selbstinterpretationen Kirchhofs Kahl, B.: Europäische Union: Bundesstaat - Staatenbund - Staatenverbund? Zum Urteil des BverfG v. 12. Oktober 1993, in: Der Staat, 33/2 (1994), 241-258.

22 Grimm, D.: Die größte Erfindung unserer Zeit, a. a. O.

23 Ders.: Braucht Europa eine Verfassung?, in: Juristenzeitung, 50/12 (1995), 581-591; erneut in: ders.: Die Verfassung und die Politik. Einsprüche und Störfälle, München, 2001, 215-254. 
sehen? Gefragt wird also nicht nach Analogien einzelner Bestandteile der bündischen Organisationen zur EU - sie gibt es, und es mag zur Analyse der EUInstitutionen hilfreich sein, sie zu betrachten -, sondern der Blick wird auf die Grundlage der EU gerichtet, ihre raison d'être.

Es ist sinnvoll, den Rückblick in die Geschichte um 1800 zu beginnen, denn die napoleonische Ära war eine erste Phase europäischer Neuordnungspolitik, in der die moderne Idee Nation entstand, wenngleich sie noch keineswegs das Denken der Menschen bestimmt hat. In dieser Zeit zerbricht mit dem Heiligen Römischen Reich Deutscher Nation ein frühneuzeitlicher Staatstyp mit einer komplexen ständischen Herrschaftsordnung, Gegenpol zum Zentralstaat frühneuzeitlicher Prägung und auch Gegenpol zum Nationalstaat der Zukunft. Was entsteht aus diesem Zusammenbruch heraus?

Das neue Europa war ein Europa der Machtkonzentration - im Staat und zwischen den Staaten. Nur die großen Staaten überlebten und diejenigen, die es schafften, sich auf Kosten anderer zu vergrößern. Europa wurde territorial umgebaut auf Kosten der Kleinen; sie gingen unter, als das Schutzdach des Alten Reiches fiel. Es gab Ausnahmen, doch die Hauptlinie hieß Machtkonzentration auf Kosten der Kleineren. Deshalb sah Jacob Burckhardt das 19. Jahrhundert dem Mysterium der Größe verfallen. ${ }^{24}$

Damit stand Jacob Burckhardt gegen einen Haupttrend europäischer Geschichte. Die Revolution, die napoleonische Expansion und dann, an Gewicht zunehmend, das große Leitbild des 19. und auch des 20. Jahrhunderts, der Nationalstaat - sie alle folgten jenen Bahnen zum zentralisierten großen Staat, die bereits der frühneuzeitliche Absolutismus eingeschlagen hatte. An diesem Punkt zielen Volksrevolution und Fürstenrevolution in die gleiche Richtung: Zentralisierung staatlicher Macht, in diesem Willen zum homogenen Staat stimmten radikale Verfechter der Volkssouveränität überein mit ihren nicht minder radikalen Gegnern aufseiten der Fürstensouveränität. Die „kleinstaatliche Massenkatastrophe“ zu Beginn des 19. Jahrhunderts, eine Formulierung des Schweizer Historikers Werner Kaegi ${ }^{25}$, ist ihr gemeinsames Werk. Dieses Zerstörungswerk legitimier-

24 Vgl. dazu Langewiesche, D.: Kleinstaat - Nationalstaat. Staatsbildungen im 19. Jahrhundert in der frühneuzeitlichen Tradition des zusammengesetzten Staates, in: ders.: Reich, Nation, Föderation, a. a. O., 194-210; ders. (Hg.): Kleinstaaten in Europa, Schaan, 2007.

25 Kaegi, W: : Über den Kleinstaat im europäischen Denken (1938), in: ders.: Historische Meditationen, Zürich, 1942, 249-314, hier 270. 
ten jene Fürsten, die es als Profiteure überlebten, auf dem Wiener Kongress. Mit ihm endete eine Phase feindlicher Übernahmen unter den Staaten Europas.

Das Europa der Dynastien legitimierte hier eine radikale Veränderung Europas, wenn auch nicht so radikal wie es zuvor Napoleon versucht hatte. Er zielte darauf, aus dem alten vielstaatlichen, ständig von Kriegen durchzogenen Europa ein europäisches Imperium zu erschaffen, das den Kontinent verwandelt hätte in einen Verbund von Staaten mit einem französischen Nationalstaat als hegemonialen Herrschaftskern. ${ }^{26}$ Das gelang nicht. Frankreich besaß nicht die Machtmittel, Europa dauerhaft zwangszuordnen. Napoleon konnte das alte Europa durch Kriege zerstören, aber nicht an dessen Stelle ein neues unter französischer Dominanz stellen.

Das konnte nicht gelingen, weil dieses neue Europa trotz Revolution, trotz Staatszerstörung und Staatserschaffung, ein Europa der Dynastien blieb. Kein neuer Staat entstand damals ohne fürstliches Haupt. Alle neuen Nationalstaaten, die bis zum Ersten Weltkrieg entstanden, wurden als Fürstenstaaten geschaffen. Ohne jede Ausnahme.

Dynastische Legitimität blieb also die dominante Grundlage von Staatlichkeit in Europa. Napoleon suchte sich ihr einzufügen. Sein europäisches Allianzsystem mit Frankreich als Kern sollte auf dynastischen Bindungen beruhen, und nicht auf dem neuen revolutionären Prinzip der Volkssouveränität. Drei Wege der Rechtssicherung seiner Kriegserfolge schlug er ein in seiner europäischen Neuordnungspolitik: Er knüpfte Heiratsverbindungen mit etablierten Dynastien, er machte Verwandte zu Königen, um einen Kranz von Satellitenstaaten zu schaffen, und er erhöhte sich zum Kaiser, um diese längst untergegangene Form von Oberheit in Europa zumindest symbolisch wiederzubeleben. Dieser Versuch, Europa um Frankreich als dominantem Kern herum zu machtpolitisch zu einen, musste schon allein deshalb scheitern, weil die beiden Hauptmächte Europas, Russland und Großbritannien, keine Nationalstaaten waren, sondern Imperien, global players, die nicht in der europäischen Arena bezwungen werden konnten.

Innerhalb Europas gilt: Der Nationalstaat löste sich von seinen revolutionären Anfängen in Nordamerika und Frankreich, er wurde zum Fürstenstaat. Der Nationalstaat als Fürstenstaat - diese Kombination wirkte als Schutzwehr gegen jede Souveränitätsteilung auf europäischer Ebene. Souveränitätssicherung, nicht Souveränitätsteilung mit anderen Staaten in einem europäischen Verbund, dieses 
Prinzip einte den jungen Nationalstaat und den historisch überkommenen Fürstenstaat. Dieses Prinzip war ihre gemeinsame Staatsräson. Die Nation und der Monarch verlangten nach ungeteilter Souveränität für ihren Staat. Auch die revolutionäre Nation. Bündische Machtteilung war ihr fremd.

Das Europa der Nationalstaaten, wie es im 19. Jahrhundert entstand und dann nach dem Ersten Weltkrieg mit der Auflösung der Habsburgermonarchie einen vorläufigen Endpunkt erreichte, schloss Souveränitätsbegrenzung zugunsten einer föderativen Ordnung oberhalb der Nationalstaaten prinzipiell aus. Die Gründe dafür sind eindeutig: Der moderne Nationalstaat zielt im Kern auf Zentralisierung und auf Machtsteigerung. Föderation hingegen bedeutet Machtteilung bis hin zum Souveränitätsverzicht zugunsten übergeordneter Institutionen. Dagegen sperrte sich die historische Idee der Nation und des Nationalstaates ebenso wie das dynastische Prinzip in der Tradition des frühneuzeitlichen Staates, der seine Staatsqualität in ungeteilter Souveränität sah. Der Nationalstaat mit fürstlichem Haupt war deshalb ein festes Bollwerk gegen jeden Versuch einer Föderalisierung der europäischen Staatenordnung.

Das gilt auch für die nationalen Föderativstaaten wie Deutschland und die Schweiz. Sie zeigten sich zwar fähig zur föderativen Dezentralisierung und damit zur Teilung staatlicher Macht, aber nur nach innen, nicht nach außen gegenüber anderen Staaten. Das zu sehen ist wichtig, um zu erkennen, wie grundlegend neu der Weg ist, auf dem sich die Europäische Union befindet. Der historische Föderativstaat hat ihr nicht den Weg gewiesen, ist nicht ihr Vorläufer. Die Organisationsform des Bundes ist es auch nicht.

Wenn nach bündischen Vorbildern für die rechtssystematische Beschreibung der EU gefragt wird, blickt man vor allem auf den Deutschen Bund. Das ist verständlich, denn dieses europäische Vertragswerk schuf in Mitteleuropa eine Staatenordnung, die fast ein Jahrhundertlang den Frieden in Europa ermöglicht hat. Es gab Kriege in Europa. Aber keinen europäischen Krieg. Aus ihm ist das Europa des Wiener Kongresses hervorgegangen, in ihm ging es unter.

Hat der Deutsche Bund ein Institutionengefüge entwickelt, das sich der Alternative Staatenbund versus Bundesstaat entzieht und deshalb analytisch aufschlussreich ist, um die EU systematisch zu bestimmen?

Gemäß der Bundesakte von 1815 vereinigten sich die ,souverainen Fürsten und freien Städte Deutschlands“ zu einem „,beständigen Bunde“. Als Zweck ihres Bundes nannten sie „Erhaltung der äußeren und inneren Sicherheit Deutschlands und der Unabhängigkeit und Unverletzbarkeit der einzelnen deutschen Staaten“. 
Darin sahen sie einen Dienst an Europa, überzeugt, dass aus ,,ihrer festen und dauerhaften Verbindung“ nicht nur die „Sicherheit und Unabhängigkeit Deutschlands“, sondern auch „die Ruhe und das Gleichgewicht Europas hervorgehen würden“. ${ }^{27}$ Die Wiener Schlussakte von 1820 definierte den Deutschen Bund dann als einen „unauflöslichen“ „völkerrechtlichen Verein“, der ,in seinem Innern als eine Gemeinschaft selbständiger unter sich unabhängiger Staaten“ besteht, ,in seinen äußern Verhältnissen aber als eine in politischer Einheit verbundene Gesammt-Macht“ ${ }^{28}$ Die Begriffe in der skizzierten rechtswissenschaftlichen Diskussion zur Analyse der EU finden sich hier also allesamt versammelt. Doch welche institutionelle Ordnung umschrieben sie damals?

Im Deutsche Bund hatten sich diejenigen Landesherren, welche das Ende des Alten Reiches überlebt hatten, zu einer dauerhaften Defensivallianz vereinigt. Defensiv nach außen und nach innen. Nach außen war das Recht des Bundes auf gemeinsames Handeln begrenzt auf „Selbstvertheidigung“ aller und jedes einzelnen Mitgliedsstaates. Allerdings nur hinsichtlich der Staatsgebiete innerhalb des Bundes. Führt ein „Bundes-Staat“, der außerbündische Gebiete besitzt, einen Krieg als „Europäische Macht“, so berührte das den Bund nicht. Defensiv auch nach innen angelegt, denn jedes Bundesmitglied verpflichtete sich, die fürstliche Souveränität nicht aufzugeben. Der Souverän darf sich im Innern durch eine „landständische Verfassung nur in der Ausübung bestimmter Rechte an die Mitwirkung der Stände“ binden lassen. Defensiv war auch das Recht des Bundes, im Notfall, wenn die Grundordnung in Gefahr war, in die inneren Angelegenheiten eines Bundesmitglieds einzugreifen. Damit wurde eine Art antirevolutionäre Gesamtverantwortung des Bundes konstituiert, nicht ein Weg eröffnet, den Bund institutionell auf Kosten der Mitgliedsstaaten auszubauen.

Dieses Gebilde Deutscher Bund zeichnen, mit Isensees Charakterisierung der EU zu sprechen, Originalität und Mutabilität aus. Es war staatsrechtlich so originell, dass schon die Zeitgenossen über seine Definition stritten und eine Vielzahl von Namen anboten: „Verein teutscher Souverainstaaten“, „freier Föderativ-Staat“, „Staatenverein“, ${ }^{29}$ andere nahmen den alten Begriff ,zusammengesetzter Staat“ auf und stellten damit den Deutschen Bund in eine lange Tradition, die in der

27 Bundesakte v. 08.06. 1815, in: Huber, E. R. (Hg.): Dokumente zur deutschen Verfassungsgeschichte, Bd. 1, 3. Aufl., Stuttgart, 1978, $84 \mathrm{f}$.

28 Ebd., 91-100.

29 Koselleck, R., a. a. O, 656-659. 
gegenwärtigen Geschichtswissenschaft wieder aufgegriffen wird $^{30}$; mehr und mehr wurde aber in der Gegenüberstellung Staatenbund versus Bundesstaat diskutiert. In welche Richtung das neue Gebilde sich entwickeln würde, hing nicht von dem Vertrag ab, sondern von den politischen Bedingungen und der $\mathrm{Zu}$ kunftsgestaltung der Akteure. Das hatte 1816 schon Wilhelm von Humboldt klar formuliert: die ,abwehrende, negativ einwirkende, Unrecht verhindernde Aufgabe“ mache ihn zum „wirklichen Staatenbund“, doch wo er als Einheit wirken wolle, werde er ,in diesen Beziehungen zu einem Bundesstaate“. 31

Wer aus diesem bündischen Gebilde eine staatstheoretische Systematik zur Definition der EU jenseits der Alternative Bundesstaat versus Staatenbund ableiten will, blendet Wichtiges aus, über das ich als Historiker nicht hinwegsehen kann:

(a) Der Deutsche Bund verfügte zwar über gemeinsame Institutionen, doch er schuf keine suprastaatliche Ebene, auf welche die Mitgliedsstaaten Souveränitätsrechte übertrugen, in deren Rahmen sie eigenständig handeln durfte. Eine Dynamik der Kompetenzerweiterung war schon gar nicht vorgesehen. Der Zollverein etwa entwickelte sich am Bund vorbei. ${ }^{32}$

(b) Im Deutschen Bund hatten sich Staaten zusammengeschlossen, die mit dem Heiligen Römischen Reich Deutscher Nation über eine lange Tradition institutioneller Gemeinsamkeiten und den damit verbundenen Souveränitätsbegrenzungen verfügte. ${ }^{33}$

(c) In diesen Staaten hatte sich ein nationales Einheitsbewusstsein entwickelt, das sich mit dem Gründungszweck des Bundes, eine dauerhafte Defensivallianz zur Sicherung des status quo nach innen und nach außen, immer weniger begnügte. Es gab eine deutsche Nation, sie organisierte sich gesellschaftlich, und sie verlangte eine Fortentwicklung des Staatenvereins in Richtung föderativer Nationalstaat mit demokratischen Partizipationsmöglichkeiten für die Staatsbürger. Ob dieser Nationalstaat mehr staatenbündisch oder mehr bundesstaatlich

30 Vor allem in der britischen Frühneuzeitforschung, ohne jedoch diese Begriffstraditionen zu kennen oder auszuweisen; vgl. mit der Literatur dazu Langewiesche, D.: Kleinstaat, a. a. O.

31 Koselleck, R., a.a.O, 658.

32 Den heutigen Stand der Forschung zum Deutschen Bund bietet Müller, J.: Deutscher Bund und deutsche Nation 1848-1866, Göttingen, 2005; ders.: Der Deutsche Bund und das politische System der Restauration 1815-1866, München, 2006.

33 Schmidt, G.: Geschichte des Alten Reiches. Staat und Nation in der Frühen Neuzeit 1495-1806, München, 1999; ders.: Wandel durch Vernunft. Deutschland 1715-1806, München, 2009. 
organisiert sein würde, hing von den machtpolitischen Verhältnissen ab. ${ }^{34}$ Diesen Schritt vom bündischen Staatenverein zum nationalen Staat, in welcher Gestalt auch immer, vollzogen die Mitgliedsstaaten nicht freiwillig. Gewalt war notwendig - revolutionäre Gewalt oder Kriegsgewalt.

\section{Europa als kollektiver Akteur in der Geschichte}

Bis in die zweite Hälfte des 20. Jahrhunderts hinein entstanden nirgendwo Instrumentarien, die es ermöglichten, bestehende Staaten in eine dauerhafte Kooperation zu bringen, indem freiwillig Souveränitätsrechte auf eine suprastaatliche Ebene übertragen und dort ein eigener institutioneller Apparat ausgebildet wird, der eigenständig staatlich handelt und darüber hinaus eine nicht mehr von den Einzelstaaten abgeleitete Legitimationsgrundlage in Gestalt eines gewählten Parlamentes erhält.

Es gab seit langem Formen kollektiver europäischer Konfliktregelung, vor allem die großen Staatenkonferenzen, auf denen völkerrechtliche Regelungen beschlossen wurden. In der ersten Hälfte des 19. Jahrhunderts kamen dann antirevolutionäre Interventionen hinzu, die der Klub der europäischen Großmächte beschloss und einer von ihnen exekutierte. Auch in der zweiten Hälfte des 19. Jahrhundert bis zum Vorabend des Ersten Weltkrieges gab es Europa als kollektiven Akteur. Doch sein Handlungsraum war nun nicht mehr Europa, hier dominierten nun die Bündnisse zwischen einem oder mehreren Staaten in einem komplizierten wechselnden Geflecht von Miteinander und Gegeneinander. Als kollektiver Akteur konnten die Großmächte Europas - die Kleinen spielten auf diesem Areopag der Staaten keine Rolle - nur noch auftreten, wenn es um Konflikte an der osmanischen Peripherie Europas ging, in der „europäischen Türkei“, wie die Zeitgenossen sagten, oder wenn sie Machtsphären außerhalb Europas absteckten, in jenem Teil der Welt, den Europa als Kolonialraum beanspruchte. Herausragende Beispiele für dieses kollektive Handeln Europas sind die Berliner Konferenzen von 1878 und 1884. Auf der ersten wurde der osmanische Balkan aufgeteilt; auf der zweiten, der Berliner Westafrikakonferenz, legten die europäischen Mächte die Spielregeln fest, nach denen binnen weniger Jahr der gesamte afrikanische Kontinent erobert und von europäischen Kolonialmächten in Besitz genommen wurde.

34 Vgl. dazu die Beiträge in: Langewiesche, D./Schmidt, G. (Hg.): Föderative Nation. Deutschlandkonzepte von der Reformation bis zum Ersten Weltkrieg, München, 2000. 
Pointiert gesagt: als kollektiver Akteur handelte Europa nur noch als globale Beutegemeinschaft und dort, wo innerhalb Europas durch ihr gemeinsames Eingreifen die Stabilität des europäischen Kontinents nicht gefährdet wurde. Als die europäischen Mächte diese Zurückhaltung in Europa aufgaben, lösten sie den Ersten Weltkrieg aus - das katastrophale Scheitern aller Versuche des 19. Jahrhunderts, Europa als kollektiven Akteur fähig zu machen, Konflikte einzudämmen.

Die Europäische Union baut ideell auf dieser leidvollen historischen Erfahrung auf, aber sie geht einen historisch gänzlich unerprobten Weg. Darin liegt ihre Originalität und die Hoffnung, die sich mit ihr verbindet. 